\title{
Controlling nanopore size, shape and stability
}

\author{
Michiel van den Hout, Adam R Hall, Meng Yue Wu, \\ Henny W Zandbergen, Cees Dekker and Nynke $H$ Dekker
}

Faculty of Applied Sciences, Delft University of Technology, Lorentzweg 1, Delft, 2628 CJ, The Netherlands

E-mail: N.H.Dekker@tudelft.nl

Received 24 December 2009, in final form 31 January 2010

Published 22 February 2010

Online at stacks.iop.org/Nano/21/115304

\begin{abstract}
Solid-state nanopores are considered a promising tool for the study of biological polymers such as DNA and RNA, due largely to their flexibility in size, potential in device integration and robustness. Here, we show that the precise shape of small nanopores $(\sim 5 \mathrm{~nm}$ diameter in $20 \mathrm{~nm}$ $\mathrm{SiN}$ membranes) can be controlled by using transmission electron microscope (TEM) beams of different sizes. However, when some of these small nanopores are immersed in an aqueous solution, their resistance is observed to decrease over time. By comparing nanopores of different shapes using (scanning) TEM both before and after immersion in aqueous solution, we demonstrate that the stability of small nanopores is related to their three-dimensional geometry, which depends on the TEM beam size employed during pore fabrication. Optimal stability is obtained using a TEM beam size of approximately the same size as the intended nanopore diameter. In addition, we show that thermal oxidation can serve as a means to independently control nanopore size following TEM fabrication. These observations provide key guidelines for the fabrication of stable solid-state nanopores on the scale of nucleic acids and small proteins.
\end{abstract}

S Online supplementary data available from stacks.iop.org/Nano/21/115304/mmedia

(Some figures in this article are in colour only in the electronic version)

\section{Introduction}

Nanopores are widely used for the detection and study of nucleic acids and other biopolymers [1-3]. In an electrolyte, such biomolecules can cause a characteristic change in the nanopore ionic conductance as they are driven through the pore by an electric field. Although a variety of different biological and synthetic nanopores have been used for such studies [1-3], solid-state nanopores, which are typically fabricated in thin membranes of $\mathrm{SiN}$ or $\mathrm{SiO}_{2}$, are the most obvious candidates for device integration and offer the largest flexibility in nanopore size and experimental conditions. A number of methods exist for the fabrication of solid-state nanopores [4-7], but the method of choice is to drill them using a transmission electron microscope (TEM) beam [6,7], as in this case the nanopore can be directly visualized during the drilling process. In this technique, nanopores are drilled by focusing a high-energy electron beam (typically $200-300 \mathrm{keV}$ ) on a thin membrane, resulting in the creation of a single pore of several nanometers in diameter. Subsequently, the nanopore can then be enlarged by continued exposure to the beam, or it can be shrunk by reducing the beam intensity $[6,7]$, allowing for very precise specification of the pore size (which is not possible using other techniques, such as focused-ion-beam sculpting). Such control is increasingly important as nanopores are used to probe complex biological systems at the molecular scale, examples of which include the unfolding of DNA hairpins [8], DNAprotein interactions [9] and, potentially, the study of RNA secondary structure $[10,11]$ and DNA sequencing [12].

Key to these applications is not only the nanopore size, but also the corresponding three-dimensional nanopore shape. For example, optimal resolution for DNA sequencing would require a very thin nanopore constriction with a local thickness on the sub-nanometer scale, so that the changes in the current blockade would reflect the presence of individual bases along the DNA. This can potentially be realized by 
fabricating a pore with a heavily compressed hourglass shape (i.e. a shape described by two mirrored cones with very wide bases). Recent tomography studies on solid-state nanopores have indicated that the shape of a nanopore depends on the material composition and precise conditions employed during the drilling, and can therefore be controlled to a certain extent $[13,14]$. Here, we demonstrate that the TEM beam size itself provides a straightforward tool to control the shape of $\sim 5 \mathrm{~nm}$ diameter nanopores drilled in $20 \mathrm{~nm}$ SiN membranes. Unexpectedly, we also find a correlation between nanopore shape and nanopore stability: a TEM beam that has a large width compared to the intended nanopore diameter (30 $\mathrm{nm}$ full width at half-maximum (FWHM) compared to a $5 \mathrm{~nm}$ diameter pore) produces nanopores with a compressed hourglass profile (figure 1(a)), but these pores are frequently observed to rapidly increase in size over time upon immersion in aqueous solutions; conversely, nanopores drilled with a narrow beam size are more cylindrical in shape, and their size is found to increase to a lesser extent, if at all. Our results indicate that, for optimal size stability, the TEM beam size should be approximately of the same size as the intended nanopore diameter. We also demonstrate control of the nanopore size following TEM drilling, showing that the size of these SiN nanopores can be gradually reduced by thermal oxidation at modest temperatures $\left(250^{\circ} \mathrm{C}\right)$, increasing their versatility even more.

\section{Materials and methods}

\subsection{Nanopore fabrication and imaging}

Nanopores were fabricated in $20 \mathrm{~nm}$ SiN membranes as previously described [15] using a Philips CM300 UT TEM, operated at an accelerating voltage of $300 \mathrm{kV}$. Pores were drilled using three different beam sizes (30, 15 and $7.5 \mathrm{~nm}$ ), where this beam size corresponds to the full width at half-maximum (FWHM) of the Gaussian intensity profile in the transverse focal plane. Scanning TEM (STEM) measurements were performed with a Cs-corrected Titan Cubed Supertwin/STEM FP5600/40 instrument operated at an accelerating voltage of $300 \mathrm{kV}$ and a beam size of $0.14 \mathrm{~nm}$. The images were acquired with a convergent angle of about $10 \mathrm{mrad}$ and camera length $184 \mathrm{~mm}$.

\subsection{Ionic current measurements}

For the electrical measurements, a microfabricated chip containing a single or multiple nanopores was first rinsed with acetone and ethanol, blown dry and exposed to $\mathrm{O}_{2}$ plasma for $30 \mathrm{~s}$. The chip was then mounted in a custombuilt Teflon flowcell [16] and a solution containing $1 \mathrm{M}$ $\mathrm{KCl}, 10 \mathrm{mM}$ Tris- $\mathrm{HCl}(\mathrm{pH}=8.0)$ and $1 \mathrm{mM}$ EDTA was introduced on both sides of the nanopore. The cis and trans sides of the nanopore are connected to separate reservoirs containing $\mathrm{Ag} / \mathrm{AgCl}$ electrodes that allow for the connection of the nanopore device to an Axon Instruments Axopatch 200B amplifier. Nanopore resistance was determined by measuring the pore current as a function of applied bias voltage and applying a linear fit to the slope of the resulting $I V$ curve using custom-written LabView software. a
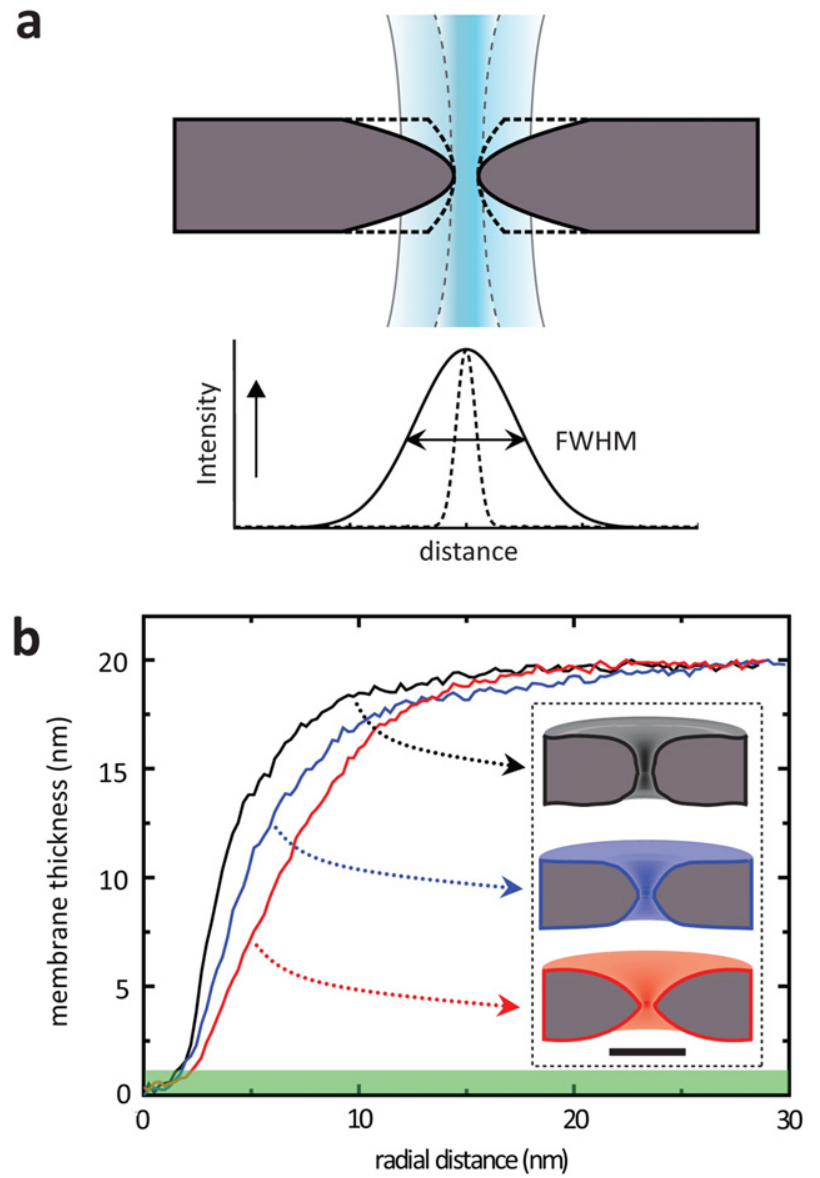

Figure 1. TEM beam size influences nanopore shape. (a) Sketch of the expected shapes of nanopores drilled with different TEM beam sizes, whose approximate 2D intensity profiles are sketched in blue. The corresponding 1D intensity profiles of the two beams are plotted below. The solid gray line delineates a TEM beam much larger than the intended pore size, whereas the dotted gray line marks a beam of approximately the same size as the targeted nanopore. The nanopore drilled with the wider beam is expected to have a compressed hourglass shape (dark gray area with solid black perimeter), whereas the nanopore for the narrower beam is expected to be more cylindrical in shape (open area with a dashed black perimeter). (b) Thickness profiles, derived from scanning transmission electron microscopy (STEM) images (section 2) of nanopores drilled with the three beam sizes ( $30 \mathrm{~nm}$, red line; $15 \mathrm{~nm}$, blue line; and $7.5 \mathrm{~nm}$, black line). (Insets) The thickness profile can be used to reconstruct a $3 \mathrm{D}$ image, assuming the nanopore is point symmetric about the center of the pore and assuming a uniform material stoichiometry. In addition, the non-zero noise in the green shaded area was removed to create the 3D images, as the TEM images indicate there is no material there. The black scale bar for the insets is $20 \mathrm{~nm}$. As the TEM beam size is increased, the nanopore shape changes from roughly cylindrical towards a compressed hourglass shape.

\subsection{Thermal oxidation}

For the experiments on nanopore oxidation, membranes containing nanopores were thermally oxidized in a Lindberg Blue Mini/Mite TF55030C- 1 tube oven, operated at $250^{\circ} \mathrm{C}$ for $1 \mathrm{~h}$ with a constant $\mathrm{O}_{2}$ flow of $10 \mathrm{sccm}$. Both before and after the oxidation, the membranes were rinsed with acetone and IPA and blown dry. 


\subsection{Image analysis}

The areas of the nanopores were computed from TEM images using ImageJ [17]. Radial intensity profiles from the STEM images were analyzed by taking a $360^{\circ}$ polar transformation (using the polar transformer plug-in for ImageJ, written by F Donnely) of the STEM image around the nanopore center and averaging line scan profiles from the center of the pore outwards. The corresponding membrane thickness was scaled such that the minimum intensity was set to 0 (corresponding to zero membrane thickness) and the maximum intensity was set to $20 \mathrm{~nm}$ (corresponding to the full membrane thickness of $20 \mathrm{~nm}$ ). A 3D image was reconstructed from these profiles by assuming cylindrical symmetry about the pore center [14]. In addition, the non-zero noise in the area at the center of the nanopore (shaded green area in figure 1(b)) was removed, as TEM images indicate that there is no material there.

\section{Results and discussion}

We first briefly explain how our nanopores are formed by the electron beam. As described in section 2, we use a focused electron beam to fabricate our nanopores in $20 \mathrm{~nm}$ thick SiN membranes. The electrons locally ablate the material, leading to the formation of a small pore. In figure 1(a) we sketch the intensity profiles for two different beam sizes and the corresponding nanopore shapes that these beams are expected to form in the SiN, based on the following reasoning: for the beam with the larger waist, a large area is exposed to a high intensity, leading to appreciable material depletion in the area around the nanopore that is formed. This will lead to the formation of a nanopore with a compressed hourglass shape (figure 1(a), solid black lines). Conversely, a beam with a smaller waist will expose only a small area around the nanopore to high intensity, giving rise to straighter sidewalls (figure 1(a), dashed black lines) and a pore that is more cylindrical in shape. Thus, we expect that, by choosing the correct TEM beam size, the nanopore shape should be controllable to some extent, which would be beneficial for many applications. For instance, a compressed hourglass shape would be particularly useful for the probing of local structure along a molecule, as the ionic resistance through the pore is dominated by the narrowest constriction; a change in the ionic conductance caused by local differences along the molecule can therefore be monitored with a resolution of the length of that constriction.

To examine whether the shape of our nanopores can indeed be controlled by using TEM beams of different sizes, we have used scanning transmission electron microscopy (STEM) to measure the shape of our nanopores. In this technique, a very small ( $0.14 \mathrm{~nm}$ FWHM) focused electron beam is scanned over the surface to create a $2 \mathrm{D}$ image. From the STEM image, a thickness profile can be derived, assuming a uniform material composition in the membrane surrounding the nanopore. A 3D shape can then be reconstructed from the STEM thickness profile by further assuming that the nanopore is point-symmetrical from its center (section 2). Previous work has shown that the shape determined in this way corresponds

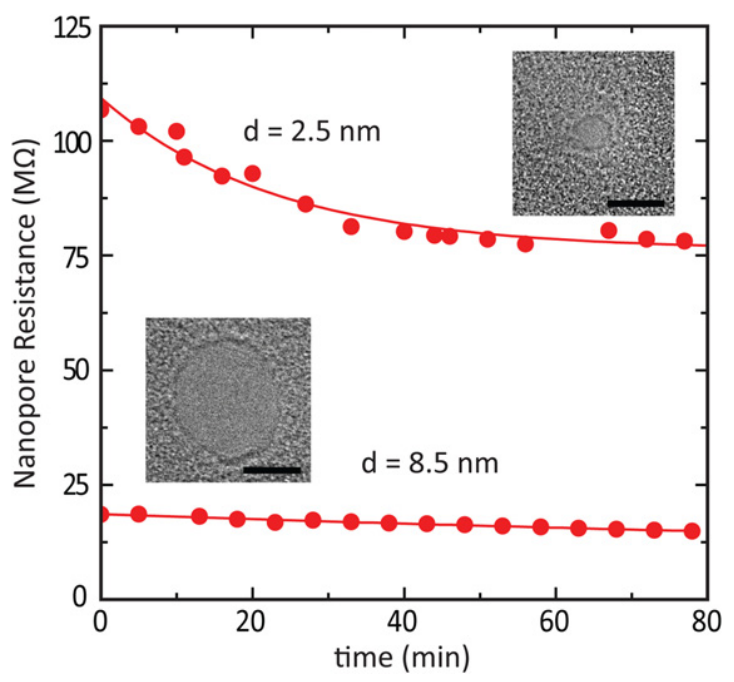

Figure 2. Ionic resistance of nanopores decreases over time. Ionic resistance (at $1 \mathrm{M} \mathrm{KCl}, 10 \mathrm{mM}$ Tris- $\mathrm{HCl}, 1 \mathrm{mM}$ EDTA, $\mathrm{pH}=8.0$ ) as a function of time of two nanopores (2.5 and $8.5 \mathrm{~nm}$, see insets), both drilled with a TEM beam of size $30 \mathrm{~nm}$ (FWHM). The solid lines are added as guides for the eyes. The scale bars in the insets correspond to $5 \mathrm{~nm}$. The ionic resistance of the small nanopore is observed to decrease strongly over the course of $1 \mathrm{~h}$, whereas that of the large nanopore decreases to a lesser extent.

well to the actual 3D structure, as determined both by TEM tomography and a number of additional analysis techniques (e.g. EELS, EFTEM) [14]. In figure 1(b), we show the radial thickness profile as determined from 2D STEM images of three different nanopores of $\sim 5 \mathrm{~nm}$ in diameter. Each nanopore was drilled with a different beam size: $30 \mathrm{~nm}$ FWHM (red line), $15 \mathrm{~nm}$ FWHM (blue) and $7.5 \mathrm{~nm}$ FWHM (black). The insets show the reconstituted 3D shapes of these pores. Indeed, the shape of the nanopore drilled with the $30 \mathrm{~nm}$ beam (red) displays the highest curvature at the center. The shape of the nanopore drilled with the smaller $15 \mathrm{~nm}$ beam is less curved at the center, whereas the nanopore drilled with the smallest beam is shown to have nearly vertical sidewalls. This demonstrates that the nanopore shape can indeed be controlled by choosing the correct TEM beam size.

We have tested our nanopores under conditions typically employed for biological experiments, in which the nanopore is immersed in an electrolyte and a constant bias voltage is applied. Surprisingly, we found that these small nanopores are frequently observed to be unstable: the ionic resistance through the nanopore continuously decreases over time during the course of an experiment, rendering such pores less useful for quantitative analysis. Examples of this behavior for two nanopores with initial diameters of 2.5 and $8.5 \mathrm{~nm}$, both drilled with a TEM beam size of $30 \mathrm{~nm}$ FWHM, are shown in figure 2. Here, we plot the time evolution of the ionic resistance through these pores over the course of $80 \mathrm{~min}$, while they were held under a constant bias voltage of $100 \mathrm{mV}$. For both pores, we observe that the resistance decreases over time. Note, however, that the effect is more pronounced for the smaller nanopore, for which the resistance drops by $26 \%$ from 107 to $79 \mathrm{M} \Omega$ in $80 \mathrm{~min}$, compared to a $15 \%$ drop from 18.5 to $15.8 \mathrm{M} \Omega$ for the larger pore. We have found that such large changes in 
a

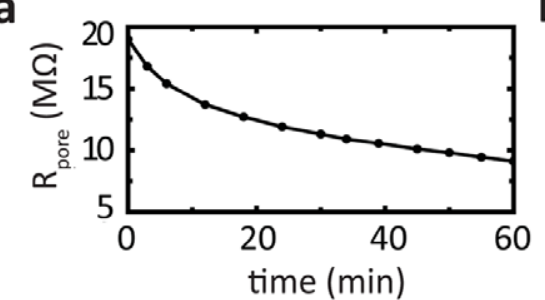

C

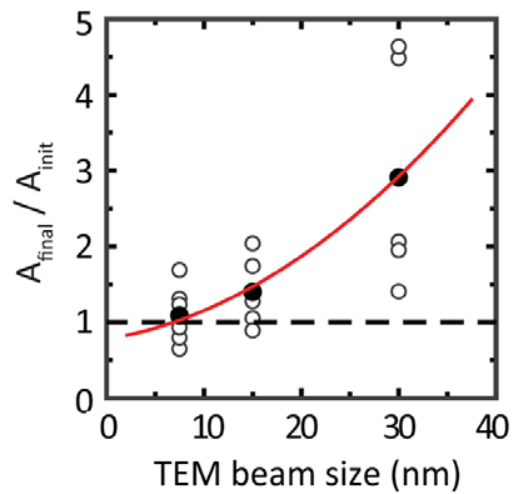

b

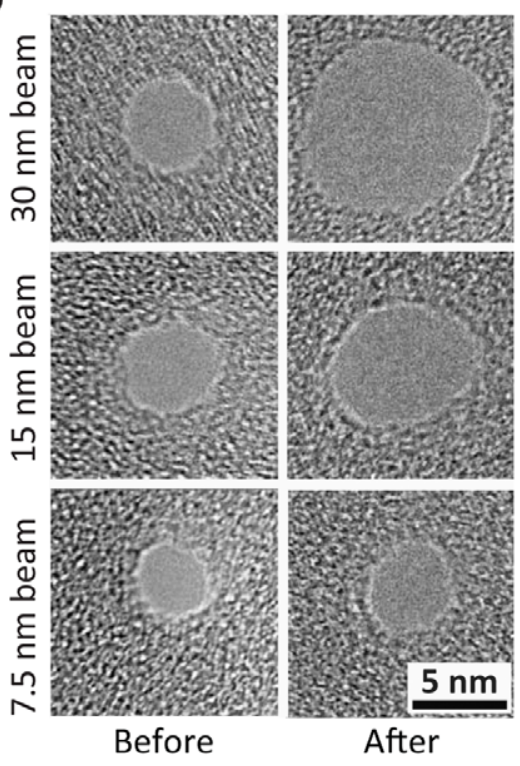

Figure 3. Nanopores drilled with wide TEM beams increase in size. (a) Resistance versus time (at $1 \mathrm{M} \mathrm{KCl,} 10 \mathrm{mM}$ Tris-HCl, $1 \mathrm{mM}$ EDTA, $\mathrm{pH}=8.0$ ) of a membrane containing multiple nanopores of approximately $5 \mathrm{~nm}$ in initial diameter, but drilled with different beam sizes. A constant bias voltage of $100 \mathrm{mV}$ is applied over the nanopores during the course of the entire measurement. The resistance is observed to decrease continuously with time. (b) TEM images of the nanopores in this membrane imaged both before (left column) and after (right column) placement in the ionic solution. The nanopore drilled with the widest beam ( $30 \mathrm{~nm}$ FWHM, top row) has become significantly larger, but this behavior is less pronounced for the nanopores drilled with smaller beams (15 nm FWHM, middle row and $7.5 \mathrm{~nm}$ FWHM, bottom row). The scale bar is $5 \mathrm{~nm}$. (c) Graph of the resulting change in nanopore (cross-sectional) area, defined as the final nanopore area divided by the initial area, as a function of the TEM beam size for different repetitions of this experiment. Open circles correspond to individual measurements and closed circles indicate the average values. The solid line is added as a guide for the eyes. The trend clearly shows that nanopores drilled with the largest beam size expand in size following immersion in an aqueous solution for $1 \mathrm{~h}$. The nanopores drilled with the smallest beam size (approximately equal to the initial nanopore size of $5 \mathrm{~nm}$ ) do not, on average, increase in size.

resistance occur most frequently for small $(<5 \mathrm{~nm})$ nanopores and, within this size range, for those pores drilled with wide TEM beam sizes ( $15 \mathrm{~nm}$ or wider). Given the fact that the TEM beam size influences the nanopore shape, this result implies that perhaps their shape is related to the instability of these nanopores.

As nanopore stability is key to the ability to perform reproducible experiments on molecule translocation, we have set out to account for the observed drop in resistance over time by examining the influence of a number of experimental parameters. First, to verify that this behavior could not be accounted for by evaporation of water from the electrolyte, we introduced fresh measurement buffer during the course of the experiment. The measurement of identical resistances before and after this operation indicated that evaporation could not underlie the observed behavior. Replacement of the $\mathrm{Ag} / \mathrm{AgCl}$ electrodes likewise did not affect the measured resistance, excluding possible electro-chemical depletion of the electrodes. A further potential explanation for the observed changes in nanopore resistance is that the nanopores themselves actually change in size over the course of a measurement in ionic solution. Indeed, even a small absolute change in diameter could have a strong effect on the resistance of a small nanopore. The effect on larger nanopores would be less pronounced, as the ionic resistance is inversely proportional to the square of the nanopore diameter [18].
Nonetheless, we note that such a size change over time is a somewhat surprising possibility, as the nanopores are drilled into $\mathrm{SiN}$ solid-state membranes, which are not expected to etch in aqueous solutions such as those used in our experiments [19].

To test whether our nanopores indeed change in size, we have compared several nanopores, drilled in a single membrane, both before and after a $1 \mathrm{~h}$ immersion in measurement buffer with an applied bias of $100 \mathrm{mV}$. Because we suspected that the size of the TEM beam used to drill the pores might be related to their stability, the sample contained three different pores with the same initial diameter $(5 \mathrm{~nm}$, see the left column in figure 3(b)), but each drilled with a different beam size (30, 15 and $7.5 \mathrm{~nm}$ FWHM). The resistance of this membrane containing three nanopores was then measured as a function of time (figure 3(a)). As before, the total resistance of the membrane gradually decreased, in this case from 18 to about $9 \mathrm{M} \Omega$ over the course of $1 \mathrm{~h}$. Images of the nanopores were taken both before (figure 3(b), left column) and after (figure 3(b), right column) the measurement, taking care to employ a very low beam intensity to ensure that the nanopores were not changed during the imaging process. Clearly, the nanopore drilled with the large $30 \mathrm{~nm}$ beam (figure 3(b), top row) increases dramatically in diameter. Similarly, the nanopore drilled with the $15 \mathrm{~nm}$ beam also increases in size, though less strongly (figure 3(b), middle row). Meanwhile, 
the nanopore drilled with the smallest beam studied $(7.5 \mathrm{~nm})$ shows very little change in diameter (figure 3(b), bottom row). This striking result implies that nanopores drilled with a wide TEM beam are inherently less stable upon immersion in an electrolyte than nanopores drilled with a narrower beam. Also, it appears that the observed change in resistance can indeed be explained by the fact that some of the nanopores changed size.

To see whether this effect of the electron beam size on the nanopore stability was reproducible, we have repeated this experiment multiple times. In each case we used the same three different beam sizes as above to produce nanopores with initial sizes ranging from 4 to $7 \mathrm{~nm}$, as determined by TEM microscopy. The results are shown in figure 3(c), where we plot the ratio of the nanopore areas before and after the experiment, $A_{\text {final }} / A_{\text {init }}$. The dashed line at $A_{\text {final }} / A_{\text {init }}=1$ is added as a reference and corresponds to no change in the area of the nanopore. Indeed, the trend of the average values confirms our initial result: pores formed with a large-diameter electron beam increase in size much more than those formed with a small beam size. The scatter in the data, especially for the large beam size, indicates that the exact size change for a given individual nanopore cannot be predicted. In some instances pores were also found to decrease in size, rather than stay constant or increase in size, and possible reasons for the observed variability will be discussed below. Yet the averages indicate a clear trend in which pore size stability decreases with TEM beam size.

It is likely that the clear difference in shape for nanopores drilled with different TEM beam sizes is related to the observed differences in stability. Our nanopores are drilled in amorphous $\mathrm{SiN}$, and it is possible that the material at the edge of the nanopores has a tendency to rearrange over time into a shape that minimizes surface tension. Sharp edges with high curvature are therefore energetically unfavorable and thus less stable. In this scenario, rearrangement is predicted to occur even in the absence of an ionic current flowing through the nanopore, as it results from energy minimization of the $\mathrm{SiN}$ only. Indeed, we have found that our nanopores also increase in size with time when stored in deionized water only, although the rate of change appears to be decreased (data not shown). Furthermore, it is also possible that the observed decrease in size for some of the nanopores drilled with the small electron beam in figure 3(c) results from a similar rearrangement: a nanopore with a highly cylindrical shape will also have sharp edges at the cylinder ends and rearrangement of these could produce the apparent decrease in diameter observed in the cross-sectional TEM image. Indeed, an additional experiment using even smaller beam sizes (2$5 \mathrm{~nm}$ ) showed a decrease in area for the pores drilled with smaller beams, whereas the pores drilled with larger beam sizes again showed a size increase (supplementary information available at stacks.iop.org/Nano/21/115304/mmedia). Thus from our two observations, namely that (1) nanopores drilled with an electron beam much wider than the nanopore size have a compressed hourglass shape and (2) such nanopores are more likely to increase in pore diameter, we can extract an important design consideration for the fabrication of nanopores: for optimal stability, a beam size of approximately the intended nanopore size is recommended.
The increasingly sophisticated control over nanopore fabrication desired for a variety of experiments can also be expanded by the ability to modify the size and surface properties of a nanopore following drilling. Here, we employ thermal oxidation as a simple tool to change the diameter of a nanopore after drilling. Upon oxidation of $\mathrm{SiN}$, nitrogen atoms are displaced by oxygen atoms, as formation of $\mathrm{SiO}_{2}$ is energetically favored over $\mathrm{SiN}$ [20]. Since the packing density of $\mathrm{SiO}_{2}$ is lower than that of $\mathrm{SiN}$, this leads to a small increase in the thickness of the material, and thus to a small decrease in the nanopore diameter, assuming a uniform material stoichiometry around the nanopore (recent results indicate that, in fact, the nitrogen content decreases close to the center of the pore [14], which would further enhance the effect of the oxidation, as pure $\mathrm{Si}$ is more rapidly oxidized than $\mathrm{SiN})$. We have investigated whether this oxidation treatment would also improve the stability of our nanopores, but no improvement was observed. Nevertheless, apart from allowing for additional size control after fabrication, thermal oxidation can have several additional advantages. For example, changing the nanopore surface into $\mathrm{SiO}_{2}$ allows for a large variety of surface chemistry reactions to further modify the nanopore surface [21]. Also, we have frequently observed that surface interactions with biological molecules are less prevalent for $\mathrm{SiO}_{2}$ than for $\mathrm{SiN}$ [22]. Such a surface modification thus also serves to increase nanopore versatility.

Thermal oxidation of thin films of crystalline $\mathrm{Si}_{3} \mathrm{~N}_{4}$ is reported to be very a slow process, with a (nonlinear) rate averaging only several $\mathrm{nm} \mathrm{min} \mathrm{m}^{-1}$ at temperatures $>900^{\circ} \mathrm{C}$ for such thin films as employed in our experiments [20]. This slow rate is useful, as it allows for very fine control over the thickness. To demonstrate that this can indeed be used to modify the size of our nanopores, we have drilled several nanopores of varying initial diameter in a single membrane (to ensure identical conditions) and thermally oxidized this membrane at $250^{\circ} \mathrm{C}$ for $1 \mathrm{~h}$ (see section 2). Such a low temperature ensures that the rate is slow enough such that even the smallest nanopores do not completely close due to the treatment. The nanopores were drilled with two different beam sizes (30 and $7.5 \mathrm{~nm}$ ), but this parameter did not appear to influence the results. In figure 4 we plot the change in area of our nanopores, again expressed as the quantity $A_{\text {final }} / A_{\text {init }}$. As expected, the area of the nanopores generally decreases by this treatment (figure 4, black points). Also, the area reduction is much more pronounced for smaller nanopores. This is not surprising, as the oxidation is expected to change the material thickness in all directions by a constant value. A $1 \mathrm{~nm}$ increase in thickness will therefore reduce the diameters of all pores by $2 \mathrm{~nm}$. Such a change yields a much more dramatic area change for small pores than for large pores. Indeed, we can fit (solid line in figure 4 ) our results well with a simple relation: $A_{\text {final }}=A_{\text {init }}\left(r_{\text {pore }}-\delta\right)^{2} /\left(r_{\text {pore }}\right)^{2}$, where $r_{\text {pore }}$ is the initial pore radius, to find an average thickness increase of $\delta=0.8 \pm$ $0.2 \mathrm{~nm}$. Comparison of our observed growth rate with reported values [20] is not possible as these were measured under very different conditions $\left(900-1100{ }^{\circ} \mathrm{C}\right.$, in partial $\mathrm{H}_{2} \mathrm{O}$ atmosphere and on crystalline $\mathrm{SiN}$, whereas our nanopores are drilled in amorphous $\mathrm{SiN}$ ). The fact that over the duration of an hour 


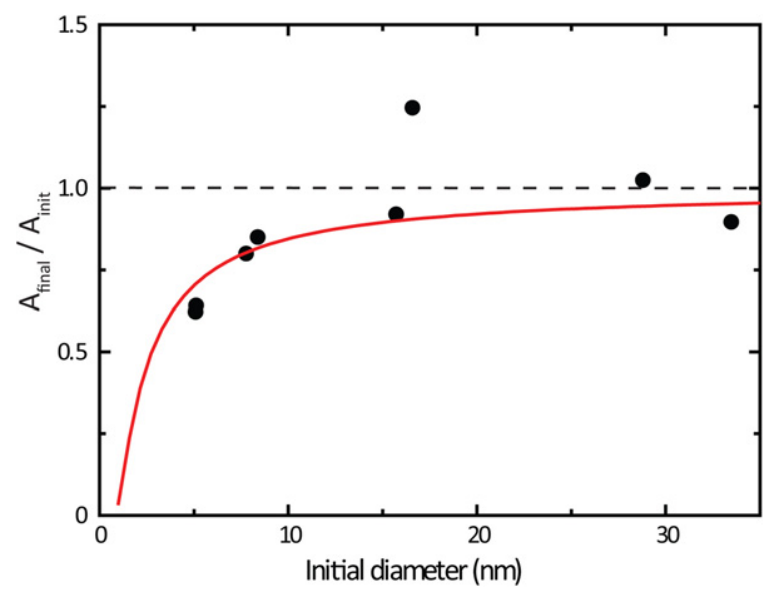

Figure 4. Nanopore size can be changed by thermal oxidation. Nanopores of different sizes were thermally oxidized at a temperature of $250^{\circ} \mathrm{C}$ for $1 \mathrm{~h}$. The ratio of the final nanopore area to the initial nanopore area is plotted as a function of the initial nanopore diameter. Almost all pores reduce in size, as expected from the fact that the oxidation of SiN should uniformly increase the membrane thickness. The solid line is a fit to the data, assuming a uniform thickness increase of the material in all directions. The fit yields a growth of $0.8 \pm 0.2 \mathrm{~nm}$, corresponding to an average decrease in nanopore diameter of $1.6 \pm 0.4 \mathrm{~nm}$.

the nanopores change only by several nanometers in diameter demonstrates that thermal oxidation offers an additional subnanometer size control for nanopores, applicable following fabrication. We also note that this technique can easily be applied at a wafer scale, making it particularly useful for applications using large arrays of nanopores.

\section{Conclusions}

We have shown that the shape of small nanopores of $\sim 5 \mathrm{~nm}$ in diameter is dependent on the TEM beam size used during their fabrication and that this influences their stability upon immersion in aqueous solution. A large TEM beam compared to the targeted nanopore size creates compressed hourglassshaped pores, which are found to increase in diameter over time during immersion in aqueous solutions. This effect is much reduced for nanopores drilled with a TEM beam size of approximately the same size as the nanopore diameter. In addition, we have shown that oxidation can further be used to modify the size of nanopores after drilling. Our results add to the understanding of nanopore formation and stability, and may serve as important design considerations for the subnanometer control of nanopore shape and size required for many of the more sophisticated applications involving solidstate nanopores.

\section{Acknowledgments}

We thank Vincent Krudde and Andrew Scott for help with electrical measurements. Financial support is acknowledged from the Netherlands Organisation for Scientific Research (NWO), Nanoned, TU Delft and from the European Science Foundation via an EURYI award.

\section{References}

[1] Dekker C 2007 Solid-state nanopores Nat. Nanotechnol. 2 209-15

[2] Healy K 2007 Nanopore-based single-molecule DNA analysis Nanomedicine 2 459-81

[3] Rhee M and Burns M A 2006 Nanopore sequencing technology: research trends and applications Trends Biotechnol. 24 580-6

[4] Chang $\mathrm{H}$ et al 2006 Fabrication and characterization of solid-state nanopores using a field emission scanning electron microscope Appl. Phys. Lett. 88103109

[5] Li J et al 2001 Ion-beam sculpting at nanometre length scales Nature 412 166-9

[6] Storm A J et al 2003 Fabrication of solid-state nanopores with single-nanometre precision Nat. Mater. 2 537-41

[7] Wu M Y et al 2005 Formation of nanopores in a $\mathrm{SiN} / \mathrm{SiO}_{2}$ membrane with an electron beam Appl. Phys. Lett. 87113106

[8] McNally B, Wanunu M and Meller A 2008 Electromechanical unzipping of individual DNA molecules using synthetic sub-2 nm pores Nano Lett. 8 3418-22

[9] Wiggin M, Tropini C, Tabard-Cossa V, Jetha N N and Marziali A 2008 Nonexponential kinetics of DNA escape from $\alpha$-hemolysin nanopores Biophys. J. 95 5317-23

[10] Gerland U, Bundschuh R and Hwa T 2004 Translocation of structured polynucleotides through nanopores Phys. Biol. 1 19-26

[11] Vocks H, Panja D and Barkema G T 2009 Amplitude and frequency spectra of thermal fluctuations of a translocating RNA molecule J. Phys.: Condens. Matter 21375105

[12] Branton D et al 2008 The potential and challenges of nanopore sequencing Nat. Biotechnol. 26 1146-53

[13] Kim M J et al 2007 Characteristics of solid-state nanometre pores fabricated using a transmission electron microscope Nanotechnology 18205302

[14] Wu M-Y et al 2008 Control of shape and material composition of solid-state nanopores Nano Lett. 9 479-84

[15] Keyser U F et al 2005 Nanopore tomography of a laser focus Nano Lett. $52253-6$

[16] Skinner G M et al 2009 Distinguishing single- and double-stranded nucleic acid molecules using solid-state nanopores Nano Lett. 9 2953-60

[17] Rasband W, ImageJ, available at http://rsb.info.nih.gov/ij National Institute of Health, Bethesda, MD

[18] Smeets R M M et al 2006 Salt dependence of ion transport and DNA translocation through solid-state nanopores Nano Lett. 6 89-95

[19] Williams K R, Gupta K and Wasilik M 2003 Etch rates for micromachining processing-Part II J. Microelectromech. Syst. 12 761-78

[20] Enomoto T et al 1978 Thermal oxidation rate of a $\mathrm{Si}_{3} \mathrm{~N}_{4}$ film and its masking effect against oxidation of silicon Japan. $J$. Appl. Phys. 17 1049-58

[21] Wanunu M and Meller A 2007 Chemically modified solid-state nanopores Nano Lett. 7 1580-5

[22] Van den Hout M et al 2010 Direct force measurements of double-stranded RNA in solid-state nanopores Nano Lett. 10 701-7 